\title{
Locking Compression Plate (LCP): Ein neuer AO-Standard
}

\author{
M. Wagner, R. Frigg
}

\section{Zusammenfassung}

Die Lockung Compression Plate (LCP) stellt nicht eine neue Platte dar, sondern ein Platten-Schrauben-System, welches die herkömmlichen Platten (T-, L-Rekonstruktionsplatten, sowie DCP und LC-DCP, etc.) ersetzen kann und zusätzlich die Möglichkeit der winkelstabilen Verankerung bietet. Die LCP liegt in den Dimensionen 3,5 Klein- und 4,5/5,0 Großfragmentsystem vor. Es steht das komplette Plattensortiment in diesem neuen Design zur Verfügung. Dank dem Kombinationsloch der LCP kann diese mit konventionellen, bikortikalen Knochenschrauben, mit Kopfverriegelungsschrauben oder mit einer Kombination von konventionellen und Kopfverriegelungsschrauben implantiert werden. Die Anwendung der LCP ist vorteilhaft bei operationspflichtigen gelenknahen Frakturen am distalen Speichenende, bei Frakturen am distalen Humerusanteil, Ellbogenluxationsfrakturen, bzw. bei proximalen Oberarmfrakturen, bei Frakturen des Schienbeinkopfes, bei proximalen Tibiafrakturen, sowie Frakturen der distalen Tibia. Vorteile sind auch bei der Stabilisierung von Osteotomien gegeben. Bei MIPPO ist die LCP auch im diaphysären Bereich vorteilhaft. Die LCP stellt einen neuen AO-Standard dar.

OP-JOURNAL 2000; 16: 238-243

(C) Georg Thieme Verlag Stuttgart · New York
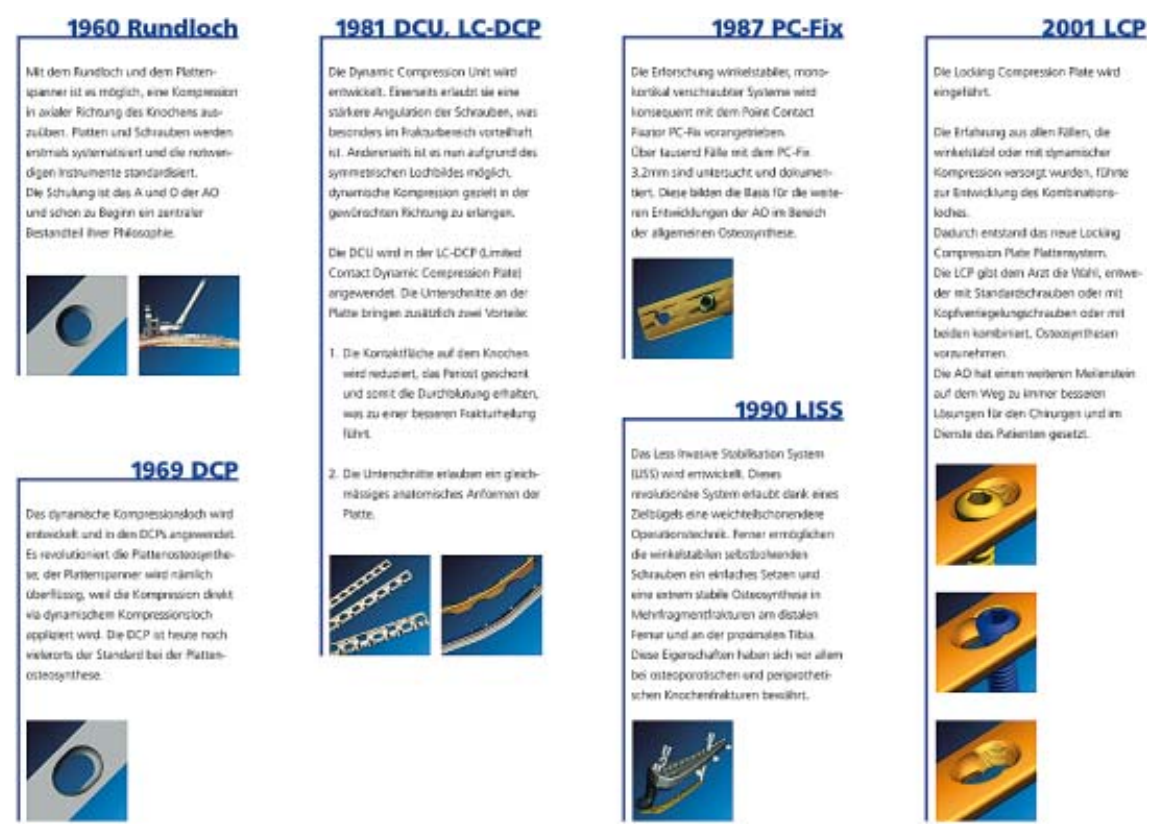

Abb.1 Meilensteine in der Entwicklung der AO-Plattenosteosynthese.

\section{Vorbemerkung}

Plattenosteosynthesen sind ein integrierter Bestandteil der operativen Frakturversorgung. Betrachtet man die geschichtliche Entwicklung der Plattenosteosynthese, so ist neben der 1960 entwickelten standardisierten AO-Rundlochplatte (gemeinsam mit dem Plattenspanner), die Entwicklung von dynamischen Kompressionsplatten „DCP“ (1969) durch die AO von großer Bedeutung. Die Meilensteine in der Entwicklung der AO-Plattenosteosynthese sind in Abb.1 ersichtlich. Um der Biologie (geringere Schädigung der periostalen Durchblutung, bzw. Reduktion der Spongiosierung des Knochens unterhalb der Platte) Rechnung zu tragen, wurde die dynamische Kompressionsplatte LC-DCP mit limitiertem Knochenkontakt LC-DCP entwickelt. Um das Problem der operativen Weichteilschädigung noch weiter zu verringern, werden in den letzten Jahren auch vermehrt minimal-invasiv perkutan eingeschobene Plattenosteosynthesen durchgeführt.
Dennoch bestehen bei der konventionellen Plattenosteosynthese mehrere Probleme:

- Die Möglichkeit eines primären Repositionsverlustes: Bei nicht ganz ideal vorgebogener Platte werden beim Festziehen der Schrauben Knochenfragmente an die Platte herangezogen und es kann zu einer Fragmentverschiebung/-kippung kommen (Abb. 2a).

- Sekundärer Repositionsverlust: Bei Frakturen mit Trümmerzonen kann es unter axialer Belastung des Knochens durch Pendeln und Auswandermöglichkeit der Schrauben im Plattenloch zu einer Fragmentverschiebung/ -kippung kommen (Abb.2b). Da die Schrauben nicht in der Platte verankert sind, können sie der Krafteinwirkung nicht entgegenwirken und werden einfach axial durch die Plattenlöcher durchgeschoben.

- Schädigung der periostalen Durchblutung durch Anpressen der Platte mittels Schrauben an den Knochen. Dieser Anpressdruck erzeugt Reibung und ge- 

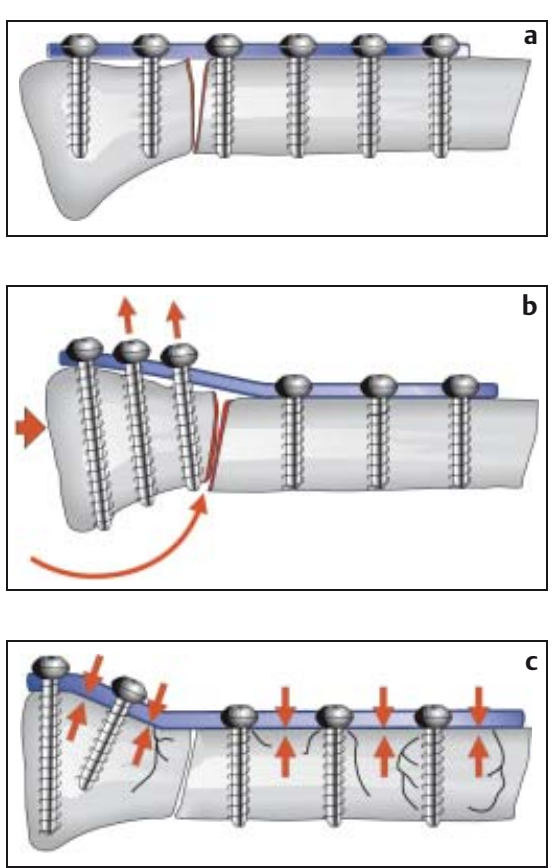

Abb. 2 Probleme der konventionellen Plattenosteosynthese. (a) Primärer Repostionsiverlust: Ist die Platte nicht anatomisch vorgeformt, dann kommt es beim Festziehen der Schrauben zu einer Fragmentverschiebung/ -kippung. (b) Sekundärer Repositionsverlust: Bei axialer Belastung des Knochens kann es durch Pendeln und Auswandern der Schrauben im Plattenloch zu einer Fragmentverschiebung/-kippung kommen. (c) Schädigung der periostalen Durchblutung durch Anpressen der Platte an den Knochen.

währleistet die erforderliche Stabilität (Abb. 2c).

Um diese Probleme zu verringern und auch die Anwendungstechnik für die perkutane Plattenosteosynthese zu verbessern, wurden winkelstabile PlattenSchrauben-Systeme entwickelt. Ein neues solches System ist besonders erwähnenswert: das Less-Invasive-Stabilisation-System für das distale Femur und für die proximale laterale Tibia (LISS DF und LISS PLT). Diese Implantate stellen anatomische, regionsspezifische Formplatten mit der Möglichkeit der winkelstabilen Schraubenfixation dar. Sie können perkutan eingebracht werden und sind aufgrund dessen in der OP-Technik anspruchsvoll.

Bei diesen winkelstabilen Platten-Schrauben-Systemen verriegeln sich die Schrauben in der Platte („Fixateur Interne“) und drücken die Platte nicht mehr an den Knochen. Die Stabilität der Frakturversorgung ist nicht mehr vom Anpressdruck, sondern von der Konstruktsteifigkeit abhängig. Deswegen werden die oben erwähnten Probleme der konventionellen Plattenosteosynthese weitgehend durch die Anwendung von winkelstabilen Platten-Schrauben-Systemen eliminiert.

\section{Wünsche und Ideen des Anwenders}

Aufgrund dieser Überlegungen wurden an die Industrie folgende Wünsche und Ideen herangetragen:

Der Wunsch des Anwenders (Operateur) ist ein winkelstabiles Implantat für die Frakturversorgung, um sekundären Repositionsverlust zu vermeiden. Dies betrifft vor allem Anwendungen der Plattenosteosynthese im Sinne einer perkutanen Plattenosteosynthese und Frakturen mit Trümmerzonen. Gleichzeitig sollte die Schädigung der Knochendurchblutung gesenkt werden. Auch bei der Stabilisierung von Osteotomien ist eine Winkelstabilität vorteilhaft und erwünscht.

Da es aber aus ökonomischen und organisatorischen Gründen nicht sinnvoll erscheint, zusätzliche neue spezielle Implantate für jede Region, bzw. Fragestellung zu entwickeln, sollten die bisherigen Platten in Form und Dimension - zumindest vorerst - belassen werden. Die herkömmlichen AO-Platten (LC-DCP mit ihrer Dynamic Compression Unit, Formplatten) sollten zusätzlich die Möglichkeit der winkelstabilen Verankerung der Knochenfragmente bieten, so dass ein variabler Einsatz von winkelstabiler und konventioneller Plattenosteosynthesetechnik gegeben ist. Ein Vorbiegen/Anmodellieren der Platte sollte möglich sein. Als weitere Bedingung wurde gefordert, dass eine möglichst geringe Anzahl von neuen zusätzlichen Instrumenten notwendig ist und das System mit den herkömmlichen Osteosynthese-Instrumenten kompatibel ist.

\section{Die Entwicklung des Kombiloches der LCP (Locking Compression Plate)}

Angespornt durch diverse Anfragen von Klinikern und speziell durch den klar formulierten Wunsch von Professor Dr. M. Wagner, eine Kombinationsplatte zu entwickeln, mit der sowohl eine Kompressionsverplattung als auch eine winkelstabile Verplattung im Sinne eines Fixateur internes möglich ist, hat sich das Entwicklungsteam an die Realisierung einer Lösung gemacht.

Die Vorgaben an das Entwicklungsteam waren klar definiert:

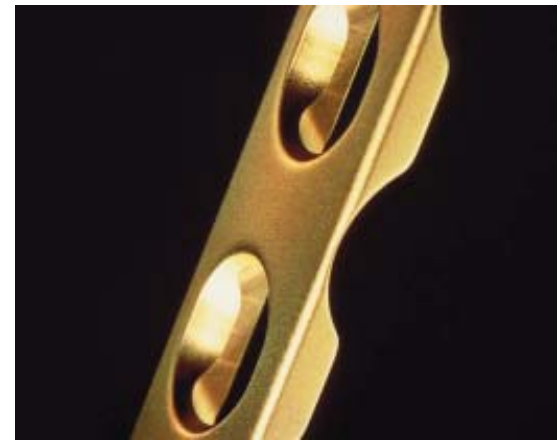

Abb. 3 Die LC-DCP und dessen DCU-Loch.

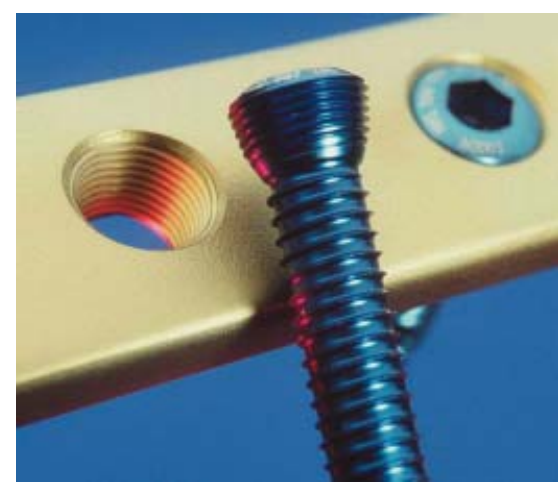

Abb. 4 Winkelstabile Verankerung bei dem LIS-System.

- Das Konzept muss für das gesamte Synthes-Plattensortiment anwendbar sein.

- Es dürfen keine Kompromisse in der Anwendung der geänderten Platten als Kompressionsplatten oder als Fixateur interne gemacht werden.

- Änderungen bezüglich Formgebung und Dimensionierung der neuen Platten dürfen nur zur Realisierung der Kombinationslöcher gemacht werden.

Gestützt auf die bisherigen Resultate der LC-DCP und dessen DCU (Dynamic Compression Unit) Plattenloch, siehe Abb.3, wurde dieses Plattenloch für den Kompressionsteil verwendet. Nicht nur die Möglichkeit der interfragmentären Kompression, sondern auch die 25-Grad-Abwinkelung der Knochenschraube in diesem Loch werden vom Kliniker geschätzt.

Zur winkelstabilen Verankerung der Schraube in der Platte hat sich das doppelgängige und konische Gewinde, wie es beim PC-Fix und LISS (Less Invasive Stabilization System) verwendet wurde, gut bewährt (Abb.4). Über 10000 solcher Kopfverriegelungsschrauben wurden im Verlaufe der letzten 4 Jahre beim PC-Fix und LISS mit Erfolg implantiert. 


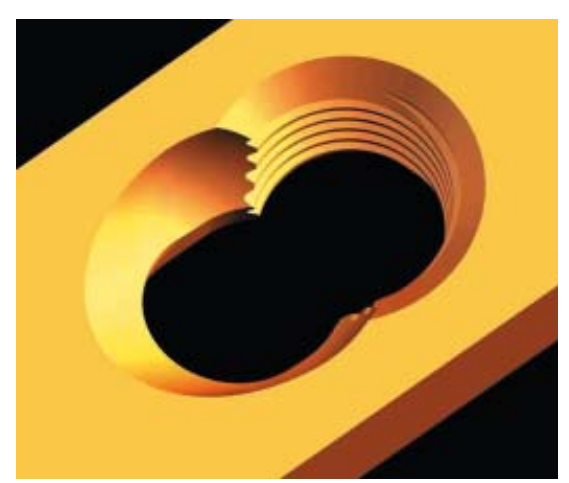

Abb. 5 Das neue Kombinationsloch.

Das nach einigem konstruktiven Aufwand entstandene Kombinationsloch aus DCU und konischem Doppelgewinde ist schlüssellochförmig (Abb.5). Das konische Doppelgewinde im Plattenloch umschließt das Schraubenkopfgewinde um über 180 Grad und liegt in der zur Plattenmitte hinweisenden Hälfte des Kombinationsloches. Der Kompressionsbereich des Kombinationsloches weist auf das Plattenende hin, wodurch eine exzentrisch gesetzte Schraube Kompression auf den Frakturspalt ausüben kann.

Neben den technischen Vorteilen, die aus der Verwendung der beiden bestehenden Plattenlöcher resultieren, sind auch die ökonomischen Vorteile nicht zu unterschätzen. Dank der Verwendung des DCU-Loches können alle zum Großfragment- und Kleinfragment-System gehörenden Bohrer, Gewindeschneider, Längenmessgeräte, Schraubenzieher, sowie Standard-Knochenschrauben der Dimensionen $3,5 \mathrm{~mm} / 4,0 \mathrm{~mm}, 4,5 \mathrm{~mm} / 6,5 \mathrm{~mm}$ und teilweise auch die durchbohrten 7,0-mm-Schrauben verwendet werden.

Vergleicht man unterschiedliche Plattenquerschnitte im Bereich des Kombinationsloches, erkennt man, dass der geringste Plattenquerschnitt im Bereich des DCU-Loches liegt. Das heißt, die mechanischen Eigenschaften z.B. einer LCDCP werden durch das zusätzliche An-

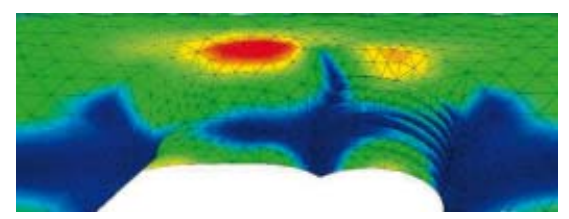

Abb. 6 Ergebnisse einer Finite-Element-Berechnung. Die höchsten Spannungen treten im DCU-Teil des Kombinationslochs auf. bringen des konischen Doppelgewindes praktisch nicht verändert. Diese Tatsache wurde im Labor und mittels Finite-Element-Berechnungen (Abb.6), neben der LC-DCP auch bei den verschiedenen Formplatten bestätigt.

Dies war für die im Projekt involvierten Kliniker, der AOTK und AO-Expertengruppen wichtig, da man an der Handhabung sprich Festigkeit, Verformbarkeit und Steifigkeit der bestehenden Platten festhalten wollte, was bei einer konstruktionsbedingten Verdickung der Platten nicht möglich gewesen wäre.

Was die Winkelstabilität der Kopfverriegelungsschraube betrifft, sind die Werte überraschend hoch. Besonders deshalb, weil der Schraubenkopf nur um ca. 240 Grad umfasst wird. Bei der LC-DCP 4,5/ 5,0 mit Kombiloch kann eine einzige Kopfverriegelungsschraube Querbelastungen in beiden Richtungen von über $1000 \mathrm{~N}$ (über $100 \mathrm{~kg}$ ) aufnehmen, bevor sie im Plattenloch abkippt. Bei dünneren Platten wie z.B. Formplatten konnte der Maximalwert der Winkelstabilität nicht erreicht werden, da sich die Platte immer plastisch deformierte (verbog) und somit das Belastungslimit setzte.

Dem Entwicklungsteam ist es gelungen, die Anforderungen an ein modernes Osteosynthese Plattensystem zu erfüllen.

Das mit Kombinationslöchern versehene neue Synthes Plattensortiment erlaubt dem Chirurgen das kompromisslose Kombinieren von Kompression und/ oder Winkelstabilität in der Plattenosteosynthese.

Die „Locking Compression Plate“ (LCP) in den Dimensionen 3,5 Klein- und 4,5/5,0 Grossfragmentsystem liegt nun vor; es steht das komplette Plattensortiment in diesem neuen Design zur Verfügung.

Das Kernstück der Ideenumsetzung ist die Entwicklung des sogenannten Kombinationsloches.

Damit können in dem LCP-Loch entweder winkelstabile Schrauben, oder herkömmliche Knochenschrauben eingebracht werden. Das LCP-Loch kann also sowohl mit Kopfverriegelungsschrauben oder mit herkömmlichen Schrauben beschickt werden. Aus biomechanischer Sicht ist bei Verwendung von Kopfverriegelungsschrauben dieses Platten-Schrauben-System einem Fixateur interne gleichzusetzen.

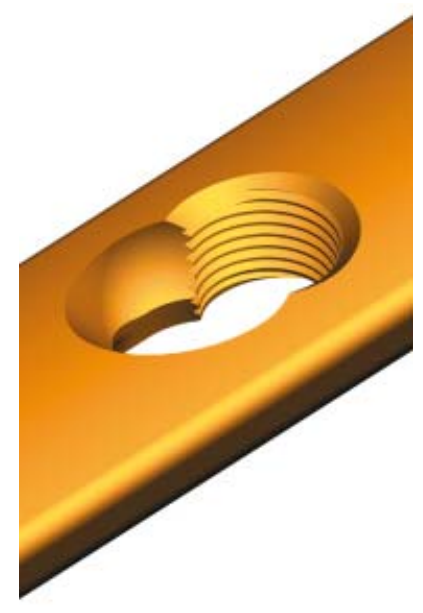

Abb. 7 Das Kombinationsloch der neuen LC Platten.

\section{Implantatbeschreibung}

Die „Locking Compression Plate“ (LCP) bietet die Möglichkeit der Kompression. Die dynamische Kompression ist wie bei den DC-Platten und im Gegensatz zu den LC-DCP nur unidirektional möglich. Das LCP-Loch ist asymmetrisch. Der Wechsel der Lochausrichtung erfolgt in der Mitte der Platte. Ein Anformen der LC-Platte ist möglich. Die LC-Platten können mit Biegeinstrumenten entsprechend den anatomischen Gegebenheiten angeformt werden.

Das LCP-Loch besteht aus zwei Teilen (Abb.7): den Lochanteil mit konischem Gewinde, welches das Verriegeln der Kopfverriegelungsschraube in der Platte erlaubt und den Lochanteil für Standardschrauben zur Ausübung dynamischer Kompression (Dynamic Compression Unit, DCU). Dieser Teil des Loches ist nicht geeignet zum Setzen einer Kopfverriegelungsschraube.

Die LCP kann mit konventionellen, bikortikalen Knochenschrauben, mit Kopfverriegelungsschrauben, oder mit einer Kombination von konventionellen- und Kopfverriegelungs-Schrauben implantiert werden.

Es gibt zwei Arten von Kopfverriegelungsschrauben (KVS): Die selbstbohrende und selbstschneidende Kopfverriegelungsschraube (Abb.8a), und die selbstschneidende Kopfverriegelungsschraube (Abb. 8b).

Die selbstbohrende und selbstschneidende Kopfverriegelungsschraube ist gekennzeichnet durch die Bohrspitze mit 

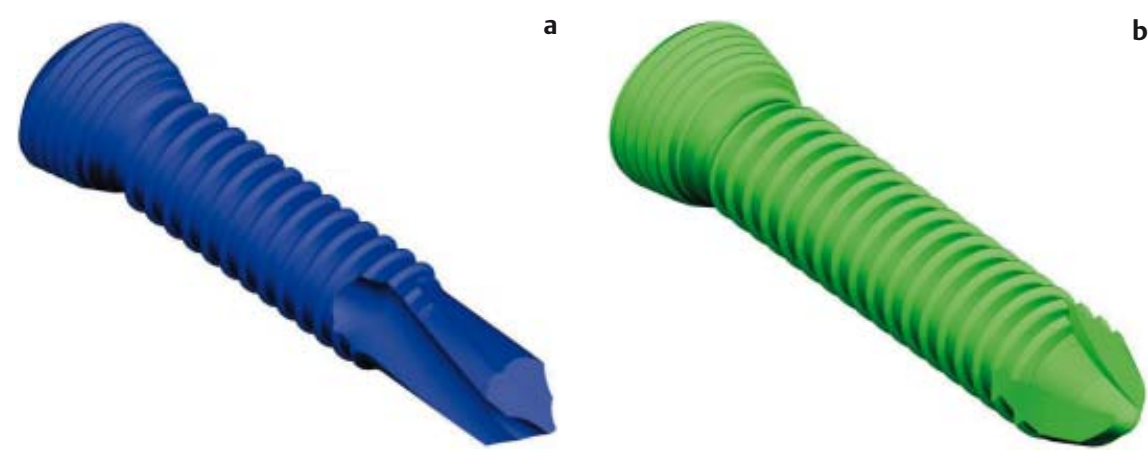

Abb. 8 Die zwei Arten von Kopfverriegelungsschrauben: (a) Die selbstbohrenden, selbstschneidende Kopfverriegelungsschraube. (b) Die selbstschneidende Kopfverriegelungsschraube.

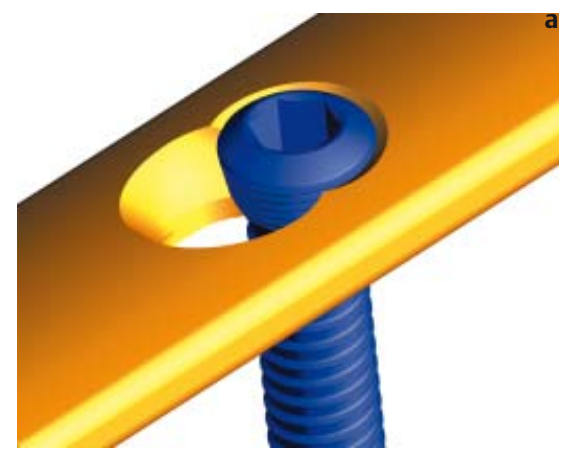

Abb. 9 LCP bietet die Möglichkeit einer Fraktur mit Standardschrauben und/oder Kopfverriegelungsschrauben zu versorgen: (a) Die Kopfverriegelungsschraube im Gewindeteil des Kombiloches. (b) Die Kortikalisschraube im DCU-Teil des Kombiloches. anschließendem Gewindeschneider, das optimierte Knochengewinde sowie das zweigängige konische Gewinde am Kopf der Schraube zur Verankerung der Schraube in der Platte.

Mit Ausnahme der Bohrspitze weist die selbstschneidende Kopfverriegelungsschraube die gleichen Eigenschaften wie die selbstbohrende und selbstschneidende Kopfverriegelungsschraube auf.

Abb. 9 zeigt eine Kopfverriegelungsschraube im Gewindeteil des Kombiloches verriegelt (Abb.9a) sowie eine Kortikalisschraube im DCU-Teil des Kombiloches (Abb.9b).

\section{Neue Instrumente zum Kleinfragmentsystem (LCP 3,5 mm)}

Nur wenige neue Instrumente sind notwendig. Die bestehenden Instrumente können bis auf die LC-DCP-Doppelbohrbüchsen weiterhin verwendet werden.

\section{Spiralbohrer $2,8 \mathrm{~mm}$}

Für die Kopfverriegelungsschrauben wird ein neuer Spiralbohrer benötigt.

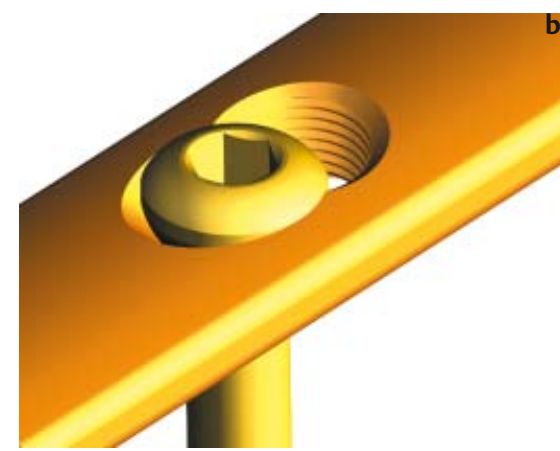

be ist besonders zu achten, da sonst die Gefahr einer Beschädigung des Innensechskants der Schraube besteht.

\section{Neue Instrumente zum Großfragmentsystem (LCP 4,5/5,0 mm)}

Die bestehenden Instrumente können bis auf die LC-DCP-Doppelbohrbüchsen weiterhin verwendet werden.

\section{Universalbohrbüchse für LCP 4,5/5,0}

Die Doppelbohrbüchse besteht auf der einen Seite aus einer 3,2-mm-Universalbohrbüchse, welche das zentrische und exzentrische Vorbohren mit einem 3,2-mm-Bohrer für die Kortikalisschrauben erlaubt. Auf der anderen Seite steht ein 4,3-mm-Bohrer zum zentrischen Vorbohren der Kortikalis für die 5,0-mmselbstbohrenden KVS zur Verfügung.

\section{Spiralbohrer $4,3 \mathrm{~mm}$}

Für die Kernlochbohrung der 5,0-mmselbstschneidenden KVS wird der 4,3-mm-Spiralbohrer verwendet.

\section{Führungsbüchse 4,3 mm, einschraubbar}

Die Führungsbüchse erlaubt ein zentrisches und orthogonales Bohren mit dem 4,3-mm-Spiralbohrer und schützt die Weichteile. Das nachträgliche korrekte Setzen der Kopfverriegelungsschrauben und deren optimale Winkelstabilität wird damit sichergestellt.

\section{Selbsthaltender Schraubenzieher-Einsatz} für 5,0-mm-KVS

Dieser Schraubenzieher-Einsatz wird zum maschinellen Implantieren der Kopfverriegelungsschrauben verwendet. Ein maschinelles Verriegeln der Schrauben muss vermieden werden, da das maximale Drehmoment der Maschinen über dem empfohlenen Anzugsmoment liegt. Das endgültige Anziehen der KVS hat deshalb mit dem Drehmomentschraubenzieher zu erfolgen.

\section{Drehmomentschraubenzieher für 5,0-mm-KVS}

\section{Schraubenzieher-Einsatz zu TLA für 3,5-mm-KVS}

Dieser Schraubenzieher-Einsatz wird in Verbindung mit dem TLA zum maschinellen Implantieren der Kopfverriegelungsschrauben verwendet. Auf den sauberen Sitz des Schraubenziehers in der Schrau-

Der drehmomentbegrenzte Schraubenzieher muss zum endgültigen Anziehen der KVS-Schrauben verwendet werden, um einerseits das benötigte Anzugsmoment garantieren zu können und andererseits einem zu starken Anziehen der KVS vorzubeugen. 


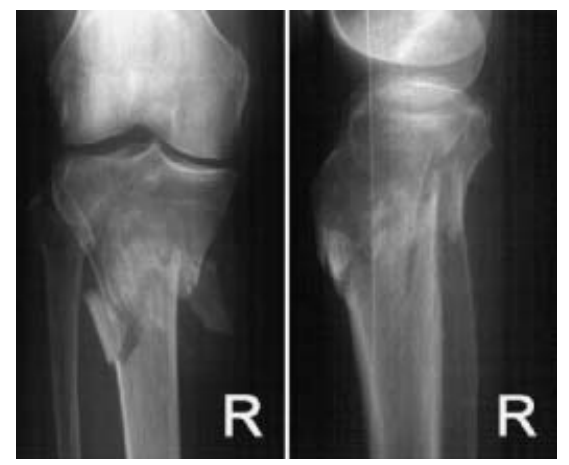

Abb.10a,b Unfallröntgenbilder einer 83jährigen Fußgängerin von PKW niedergestoBen, prox. Tibiafraktur mit schweren Weichteilschaden an der lateralen Seite des Unterschenkels (AO-Klassifikation 41-C2).

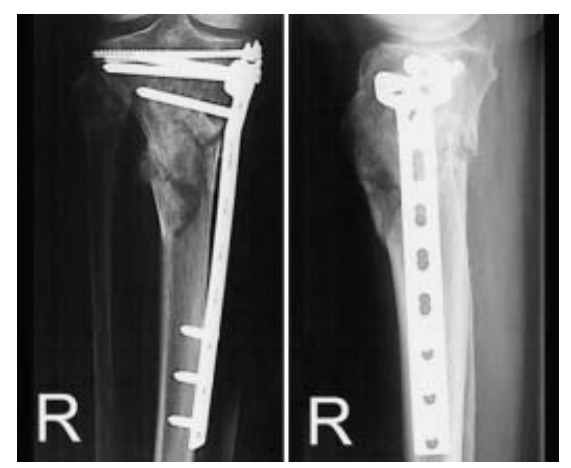

Abb.10c, d Postoperative Röntgenkontrolle: Verschraubung der interartikulären Fraktur mit 24,5 kanülierten Zugschrauben, Überbrückung der Metaphysären Trümmerzone mit einer 8-Loch-LCP-T-Platte und monokortikalen winkelstabilen Schrauben.

\section{Die Frakturversorgung mit der LCP zeichnet sich durch folgende Punkte aus:}

- Es ist möglich, Frakturen wie bisher mit herkömmlichen Spongiosa- oder Kortikalisschrauben zu versorgen.

- Die LC-Platte kann ausschließlich mit Kopfverriegelungsschrauben eingesetzt werden, wodurch die Platte zum reinen internen Fixateur wird. Ein interner Fixateur verhält sich aufgrund seines Wesens anders als ein konventioneller Schrauben/PlattenAufbau.

a Die LC-Platte kann mit einer Kombination von herkömmlichen Schrauben und Kopfverriegelungsschrauben fixiert werden.

\section{Anwendung der LCP in der Klinik:}

Ein erster Handling-Test in den Anwender-Kliniken verlief erfolgversprechend

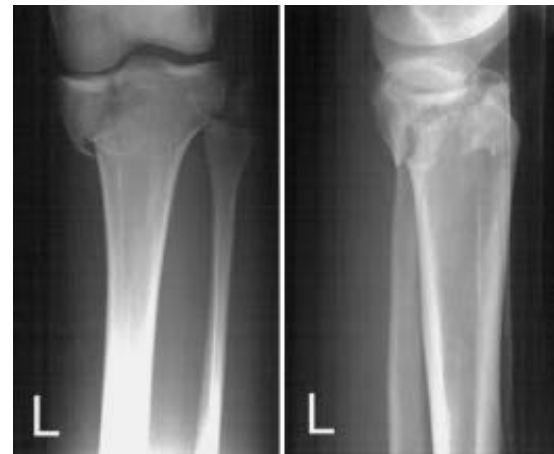

Abb.11a,b Unfallröntgen 21-jähriges Mädchen Reitunfall, Schienbeinkopf/Luxationsfraktur (AO-Klassifikation 41-C3).

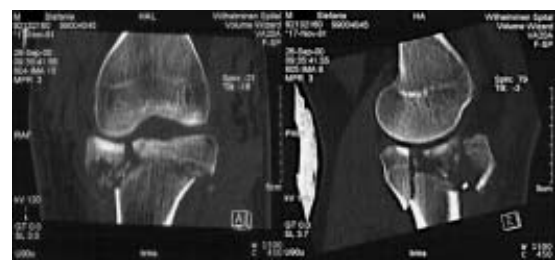

Abb.11 c, d Computertomographische Darstellung der intraartikulären Mehrfragmentfraktur.

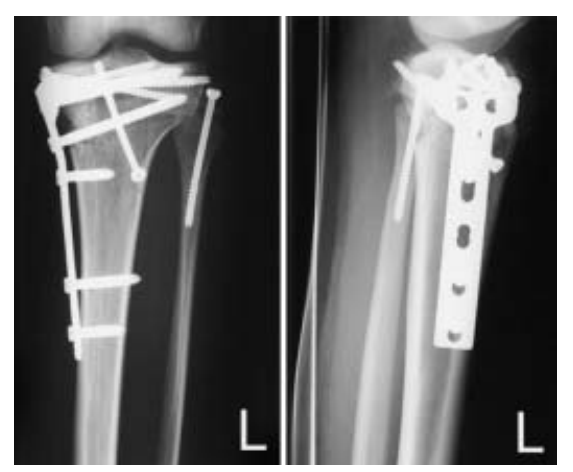

Abb.11 e,f Postoperative Röntgenbilder, Frakturversorgung mittels Zugschrauben und medial eingeschobener 5-Loch-LCP-TPlatte, versorgt. Der knöcherne Abriss des äußeren Seitenbandes wurde mit einer Zugschraube und Zuggurtung versorgt.

und hinterließ einen sehr positiven Eindruck.

Aus heutiger Sicht ist ihre Anwendung vorteilhaft bei operationspflichtigen gelenknahen Frakturen am distalen Speichenende, bei Frakturen am distalen Humerusanteil, Ellbogenluxationsfrakturen bzw. bei proximalen Oberarmfrakturen, bei Frakturen des Schienbeinkopfes, bei proximalen Tibiafrakturen sowie bei Frakturen an der distalen Tibia. Große Vorteile sind bei der Stabilisierung von Osteotomien gegeben. Bei MIPPO ist die LCP auch im diaphysären Bereich vorteil-

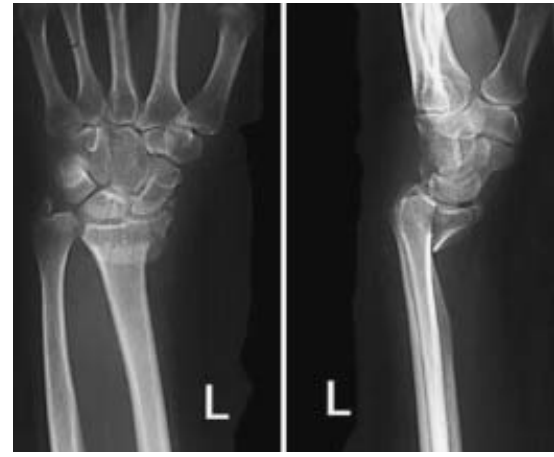

Abb.12a,b Unfallröntgenbilder einer dist. Speichenfraktur (AO-Klassifikation 23-C3) bei einer 59-jährigen Patientin.

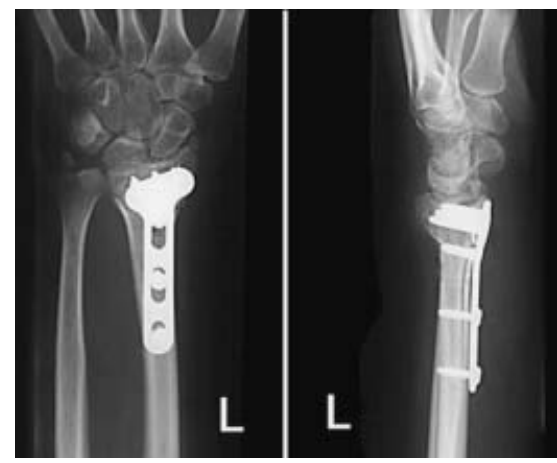

Abb.12c,d Postoperative Röntgenbilder, Frakturversorgung: mittels volarer Plattensynthese mit winkelstabiler LCP-T-Platte 3,5 schrägwinkelig, gipsfreie frühfunktionelle Nachbehandlung.

haft. Ob Vorteile bei indizierter Plattenosteosynthese von Unterarmschaftfrakturen gegeben sind, muss erst die Erfahrung zeigen.

Die Abb.10, 11 und 12 zeigen drei klinische Beispiele für den Einsatz der LC-Platte.

Vorteile des neuen Platten-SchraubenSystemes (LCP) aus der Sicht des Anwenders:

- die Winkelstabilität und die daraus resultierende bessere Verankerung

- die Platte, wenn die mit Kopfverriegelungsschrauben angewendet wird, muß nicht exakt an den Knochen anmodelliert werden

- geringe Schädigung des Periostes, somit bleibt die Durchblutung erhalten, was zu einer besseren Frakturheilung führt

a geringe Möglichkeit der Schraubenlockerung, da diese sich mit ihrem Schraubenkopf im Gewindeanteil des Plattenloches verklemmen 
einfache Technik

die neue Technik zeichnet sich unter anderem durch die anwenderfreundliche Verwendung von selbstbohrenden und selbstschneidenden Kopfverriegelungsschrauben, welche nur monokortikal eingebracht werden müssen, aus. Die Arbeitsschritte Vorbohren, Länge messen und Gewinde schneiden können entfallen, das Einbringen der Schraube ist mit maschinellem Antrieb möglich und sinnvoll, lediglich die definitive Fixation erfolgt mit einem Drehmomentschraubenzieher. Diese monokortikalen Schrauben beeinträchtigen die Durchblutung weniger.

- die Möglichkeit der Standardplattenoperationstechnik mit interfragmentärer Kompression über die Platte mit Hilfe von konventionellen Schrauben im Lochanteil für dynamische Kompression (Dynamic Compression Unit DCU) und das Einbringen einer Zugschraube durch die Platte

- die Platte kann vorgebogen werden; die Unterschnitte erlauben ein gleichmässiges anatomisches Anformen die LCP ist eine limitierte Kontaktplatte und hat dadurch einen reduzierten Kontakt zum Knochen, auch bei Anwendung der Standardplattentechnik. Überdies ist die Platte mit Unterschneidungen an der Unterfläche ausgebildet, wodurch ein evtl. Anpressdruck an das Periost nur punktuell erfolgt

das System ist kompatibel mit existierenden Osteosynthese-Instrumenten und konventionellen Knochenschrauben

nur wenige zusätzliche Instrumente sind notwendig

Bei der LCP handelt es sich nicht um eine neue Platte, sondern um ein PlattenSchrauben-System, welches die herkömmlichen Platten (T-, L-, Rekonstruktionsplatten sowie DCP und LC-DCP, etc.) ersetzen kann und zusätzlich die Möglichkeit der winkelstabilen Verankerung bietet.

Die LCP stellt einen neuen AO-Standard dar.

\section{Literatur}

${ }^{1}$ Krettek C. Distale Femurfrakturen (perkutane Plattenosteosynthesen). Unfallchirurg 1996; 99: 2-10

2 Nünninghoff U, Hierholzer G. Geschichte und Entwicklung der Plattenosteosynthese. OP-Journal 1997; 13: 232-235

3 Perren S. Grundlagen der Plattenosteosynthese - Biologie und Biomechanik. OP-Journal 1997; 13: 238-246

4 Rüedi TP, Murphy WM. AO Principles of Fracture Management. Georg Thieme Verlag, Stuttgart, New York 2000

5 Rüter A, Trentz O, Wagner M. Unfallchirurgie. Urban \& Schwarzenberg, München, Wien, Baltimore 1995

\section{Prof. Dr. med. M. Wagner}

Abteilungsvorstand

Unfallchirurgische und sporttraumatologische Abteilung des Wilhelminenspitals der Stadt Wien Montleartstraße 37

A-1160 Wien

Österreich

\section{R. Frigg}

Leiter Neue Konzepte

Mathys Medizinaltechnik AG

Güterstraße 5

CH-2544 Bettlach

Schweiz 